\title{
Parameterising the Dynamics of Inter-Group Conflict from Real World Data
}

\author{
Liam D. Turner*, Gualtiero B. Colombo*, Roger M. Whitaker*, Diane Felmlee ${ }^{\dagger}$ \\ ${ }^{*}$ School of Computer Science \& Informatics, Cardiff University, UK \\ \{TurnerL9, ColomboG, WhitakerRM\}@ cardiff.ac.uk \\ ${ }^{\dagger}$ Dept. of Sociology \& Criminology, Pennsylvania State University, PA, UK \\ dhf12@psu.edu
}

\begin{abstract}
Generative modelling of inter-group relations enables probabilistic forecasting of possible conflict for scenarios where real-world data is sparse. In order for such models to have relevance and integrity, it is important to ensure that realworld data is used to parameterise the model and verify its characteristics. In this paper we investigate how real-world datasets can be mapped into generative model parameters concerning group structures and behaviours. We highlight the issues involved and present a framework for classifying potential data based on three attributes: (i) inter-group structure, (ii) inter-group actions and (iii) impact of actions. We argue that these attributes are fundamental for benchmarking and developing generative models in the context of limited existing data on inter-group interaction.
\end{abstract}

\section{INTRODUCTION}

A major concern in asymmetric warfare is the threat faced by coalition operations, originating from insurgent group activity [1], [2]. In many cases such groups are loosely and chaotically organised, but their ideals are sociologically and psychologically embedded across members such that the group has expected behaviours that can represent a major threat. Therefore understanding how groups change, interact and conflict in different situations is of significant interest [3], [4], [5]. However it is frequently the case that limited pre-existing data is available from which techniques such as machine learning can be employed to make predictions of the future based on past events. As such, alternative approaches to gain insights into possible future behaviour are needed.

In this context generative modelling is a promising approach which has gained considerable interest for studying social phenomena [6]. Frequently embodied through agent-based computation [7], this involves a model of human behaviour, often with a stochastic component (e.g., [1]), that can be parameterised to forecast the emergence of future events for particular scenarios. Agent-based modelling is often adopted because event-driven interactions allow collective phenomena to emerge, such as cooperation or hostility, which are important to social observations and can be challenging to gain through other approaches.

Developing models of this nature is complex as it involves emulating human behaviour (e.g., [8], [4], [9]). To make progress, models need to be grounded in relevant psychological and sociological theory that can be translated to a quantitative representation. Furthermore, for modelling to support

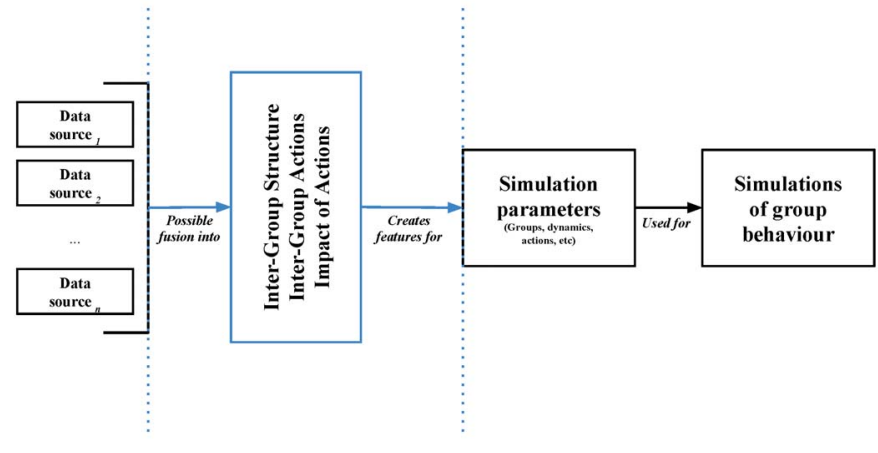

Fig. 1. Schematic representation of parameterising generative models (outlined in blue) from real-world data.

decision making, there needs to be accurate representation of a physical scenario, and confidence in the model's ability to generate "real-world" behaviours needs to be examined.

In this paper we tackle both these issues by considering the use of existing datasets in parameterising and testing generative models. Due to the heterogeneous and often sparse nature of data, this is a non-trivial task that has received limited attention in the literature. We identify a number of potential datasets that capture conflicts and use one in particular to exemplify a proposed framework to parameterise the dynamics of inter-group conflict (visualised in Figure 1). We argue that this necessary step will allow better adaptation and verification of generative models, increasing their integrity and the support they can provide for decision-making in military and peacekeeping environments. Importantly, this approach supports commonality of analysis in multi-organisation federations such as military coalitions.

\section{FRAMEWORK}

Due to the diversity of data available on conflicts, a common framework for organising data extraction is beneficial. There are wide-ranging potential datasets available (see Section IV) that exist with limited cross-dataset conventions on coding, formatting, and data integrity. Furthermore data exists for a variety of different conflicts that are distinguished by scale, actors and wider context. Therefore it is important to deploy a common approach to extracting relevant features from datasets. 
Importantly this enables different datasets to be integrated, permitting data fusion that can enrich scenario planning.

The framework we propose is motivated by the widely used approach of classifying behaviour by asking who, what, when, where and how? Variants of this framework are widely used, typically examining individual actors and scenarios. It is therefore necessary to translate this to a form that can be used for inter-group situations, potentially where there are many events and multiple groups. Our proposal for achieving this identifies three fundamental components that need to be considered: These concern: (i) inter-group structure, (ii) intergroup actions and (iii) impact of actions. We consider each of these in turn.

\section{Inter-Group Structure}

Inter-group structure concerns the number of identifiable groups as entities that form the basis of modelling. In many cases these may be difficult to explicitly define, subject to rapid change and may overlap, but these present the primary entities between which interaction should be considered. This follows the direction of Reicher [10], where groups represent the collective identity of individuals. Derivation of social identity through groups [8] plays a significant role in intergroup conflict: under situations of heightened tension the identity of the individual is diminished and group identity takes precedence.

Social network analysis has been a well-used methodology applied to the internal structure of groups that have been featured in conflict and terrorism situations [11], [12]. When considering inter-group conflict, the relations between rather than within groups are of primary interest. Edges between groups can be used to represent a range of issues, primarily focussed on the extent of inter-group actions.

\section{Inter-Group Actions}

Inter-group actions represent individuals acting on behalf of groups, towards another group (or groups). These may directly feature in event-driven models, such as those which are agent based, giving a direct link between data sources and generative modelling. The time, location and frequency of actions are fundamental components of conflict situations, and usually form a key part of associated datasets.

As such these actions document the dynamics that lead to escalation of conflict. Therefore classifying the temporal nature of actions, and their form, is important. Actions may also have geographical relevance and can be represented in a static (as well as dynamic) form using edges between associated groups. Reduction of datasets into a coded form, using an ontology, is useful practice.

\section{Impact of Actions}

The impact of actions concerns interpreting the nature of specific events that are targeted at groups. These can be represented on a continuum between conflict and cooperation. The type of the event that impacts receiving group(s) is an influential factor in conflict escalation and related strengthening of group identities.
Therefore using inter-group structure, inter-group actions and impact of actions allows categorisation that is useful in systematically characterising data from multiple sources, and consolidating data entry issues. This provides a basis to parameterise a generative model, defining the scope of actions, for example in terms of the costs to a receiving group.

\section{APPLICATION OF THE FRAMEWORK}

There are two ways in which data concerning inter-group structure, inter-group actions and the impact of actions can be used to support the integrity of generative modelling. The first concerns scenario building, where it is necessary to ensure that the characteristics of the scenario are properly embedded in the model. This involves ensuring that the presence of different groups (e.g., number of groups and their characteristics), the potential types of actions they perform and the consequences (e.g., the costs to the receiving group) are sufficiently embedded in the model. These three areas are model independent, and should be considered irrespective of the underlying theoretical approach.

The second issue concerns consistency between the generative model and "real-world" data as represented through the framework. This involves exploring to what extent implementation of the particular generative model can lead to inter-group actions and impact on groups in a manner that is consistent with these datasets. In should be noted that inconsistency between the model and a dataset does not necessarily imply that the model is "incorrect". The data on which the model is based is likely to be subject to noise and omission, while at the same time the theory underpinning the model is a reductionist view of human behaviour.

However it is appropriate to test the assumptions of the model by exploring whether the model can be parameterised such that its output has features consistent with the data present in the framework that captures the real world. In such a case and assuming that the data present in the framework is accurate, the model provides an instance where the underpinning theory is aligned with external events. Clearly the greater the extent of such instances being based on realworld data, the greater confidence can be had in the overall generative approach. We note that some generative models may be governed by a large input parameter space. This may result in a complex tuning problem, based on finding combinations of input parameters that provide output data consistent with the real world.

\section{DATASETS}

In this section we present examples of candidate datasets that describe different inter-group conflict scenarios. Datasets that are relevant to group conflict often provide limited utility due to common issues including sparseness of the data, as well as data quality issues arising from being encoded by human annotators from qualitative data. This presents a challenge when benchmarking generative models, which can be overcome to some degree by looking to combine multiple datasets for the same scenario where possible. 
TABLE I

COMPARISON OF REAL-WORLD DATASETS AND THERE RELEVANCE TO OUR INTER-GROUP CONFLICT FRAMEWORK. IGS=INTER-GROUP STRUCTURE, IGA=INTER-GROUP ACTIONS, IOA=IMPACT OF ACTIONS. CLASSIFIED RELEVANCE OF FEATURES: $\bullet=$ STRONG, $\circ=$ MODERATE, $-=$ LIMITED.

\begin{tabular}{|c|c|c|c|c|c|c|c|c|}
\hline Dataset & Description & Locality & Period & \# Events & Features & IGS & IGA & IOA \\
\hline SCAD & $\begin{array}{l}\text { The Social Conflict Analysis } \\
\text { Database [13] datasets contain } \\
\text { social conflicts including riots and } \\
\text { strikes. Version } 3.2 \text { is used. }\end{array}$ & Africa & $1990-2015$ & 14,702 & $\begin{array}{l}\text { Temporal bounds, actors in- } \\
\text { stigating conflict, targeted ac- } \\
\text { tors receiving conflict, involved, } \\
\text { type of conflict, type of esca- } \\
\text { lation, number of participants, } \\
\text { government repression, issues } \\
\text { seen as cause of the conflict, } \\
\text { location, fatalities, source of in- } \\
\text { formation. }\end{array}$ & - & • & ○ \\
\hline ACLED & $\begin{array}{l}\text { The Armed Conflict Location and } \\
\text { Event Data Project [14] provides } \\
\text { disaggregated data of conflicts } \\
\text { spanning multiple geographic re- } \\
\text { gions. The Africa dataset explored } \\
\text { is version 7, The Asia dataset ex- } \\
\text { plored include the combined "Re- } \\
\text { altime Running Asia Files", until } \\
\text { April } 2017 \text {. }\end{array}$ & Africa & $1997-2016$ & 140,746 & $\begin{array}{l}\text { Temporal bounds, actors in- } \\
\text { volved, type of conflict, loca- } \\
\text { tion, fatalities, source of infor- } \\
\text { mation. }\end{array}$ & - & $\bullet$ & - \\
\hline UCDP GED & $\begin{array}{l}\text { The Uppsala Conflict Data Pro- } \\
\text { gram datasets [15] capture world- } \\
\text { wide organised violence and con- } \\
\text { flict. The dataset explored is ver- } \\
\text { sion } 5 \text { of the Georeferenced Event } \\
\text { Dataset (GED), however the pro- } \\
\text { gram also offers multiple different } \\
\text { datasets and subsets. }\end{array}$ & Worldwide & 1989-2015 & 128,264 & $\begin{array}{l}\text { Temporal bounds, actors in- } \\
\text { volved, type of conflict, loca- } \\
\text { tion, fatalities, source of infor- } \\
\text { mation. }\end{array}$ & - & - & - \\
\hline UCN & $\begin{array}{l}\text { The UCINET Covert Networks } \\
\text { datasets are standalone datasets as- } \\
\text { sociated with the UCINET soft- } \\
\text { ware package [15]. They contain } \\
\text { social network data of groups span- } \\
\text { ning either individual events or ex- } \\
\text { tended time periods. }\end{array}$ & Various & Various & Various & $\begin{array}{l}\text { Varies per dataset, mostly the } \\
\text { social network of individuals. }\end{array}$ & o & - & \\
\hline
\end{tabular}

\section{Examples}

Several popular and substantive datasets of conflict exist in the literature [16], including: SCAD [13], UCDP [15], ACLED [14], and the UCINET covert networks (UCN) datasets from the UCINET software package [17]. These datasets can be comprised of multiple individual sub-datasets (e.g., UCN), or additional, more focused subsets a larger dataset (e.g., UCDP). Additionally, further related datasets extend some of these with additional semantic detail (e.g. location) [18] or temporal extensions [19]. Across these, the scope of individual datasets can vary, by either focusing on conflict events worldwide (e.g., UCDP) or at some particular location (e.g., Africa [13]), and in the the types of conflicts included, with some providing a more holistic view (e.g., SCAD), and others focusing on a particular type, e.g. PRIO Battle Deaths [20]. Collectively, these datasets have been involved in extensive research into trends of conflict across the world (e.g., [21], [22], [23]), but to the best of our knowledge, they have not been used as a basis for creating generative models of group conflict.

The information captured in these datasets also vary, with some focusing on the properties of the entities involved in the conflicts, or relationships between them (e.g., UCN), and others focusing on details surrounding the events that occur, such as the parties involved and the location (e.g., ACLED, SCAD). Table I classifies these datasets by how the data contained spans the three areas of generative models of group conflict outlined in Section III, with the meta-analysis showing that the scope of individual datasets do not provide comprehensive coverage.

Among these datasets, SCAD and UCDP's GED dataset provide the broadest coverage (as visualised in Table I), however they only provide limited detail surrounding the entities involved in the conflict events, and the impact and fallout of actions. However, this limitation is likely reflective of the challenges in capturing this information, where the properties and dynamics of groups may not be transparent and externally observable. This issue of breadth in individual datasets creates the primary challenge of using this data for scenario generation, aside from the sparseness and data quality issues, with interconnectivity between datasets also often limited.

A second issue of these datasets is that the events can 
TABLE II

CHARACTERISTICS OF UNIQUE GROUPS EXTRACTED (N=9110).

\begin{tabular}{lr}
\hline Characteristic & \# Groups \\
\hline Type of group & 1530 \\
State & 7580 \\
Non-state (or unknown) & \\
\hline Passiveness: Proportion of actions performed/received & 3873 \\
Always recipient of conflict & 4414 \\
Always instigator of conflict & 823 \\
Both instigator and recipient & \\
\hline Cooperativeness: How often groups act together & 1091 \\
Always cooperative & 7276 \\
Never cooperative & 743 \\
Sometimes cooperative & \\
\hline
\end{tabular}

involve individuals as the source or recipient of the action, however these cases may not be distinguishable without human interpretation, and that the groups may not be organised groups, but also spontaneous collections of individuals.

\section{CASe Study: SCAD Africa v3.2 Dataset}

To understand how a real-world dataset can be considered in terms of inter-group structure, inter-group actions and impact of actions, we present a case study focussed on the SCAD Africa v3.2 dataset [13]. Notably this dataset contains data sufficient to span all three features. The dataset contains 14,702 events that represent actions from one set of groups to another, however 3863 were removed due to either being duplicate entries for different locations or placeholder entries (as defined by the dataset's codebook).

The results of this case study demonstrate how the conflict dataset can be used to parameterise generative models. Importantly the case study highlights that the event-based format of this data set results in the need to post-process the dataset to extract features that are relevant to generative models. The components of the proposed framework support this.

\section{A. Inter-Group Structure}

The SCAD dataset primarily focuses on the characteristics of individual conflicts, providing limited detail concerning the parties involved. As a result, details surrounding the structure of individual groups (i.e., size, leadership, connectedness) and their members are not provided. However, where the groups involved are distinguishable (e.g., "Al-Qaeda", rather than "Youths"), this information can potentially be supplemented by using additional datasets.

Whether the recipient of an action is a governmental (state) group can be directly determined from the dataset, and additional characteristics can be inferred from examining patterns in the actions that particular groups perform. Some examples of these include the passiveness of a group (through whether a group is always the recipient of conflict, the source, or both), and the cooperative nature of a group (through whether they always, sometimes, or never cooperate with another party when conducting an action). Table II shows the distribution of unique groups in the dataset for these characteristics (after

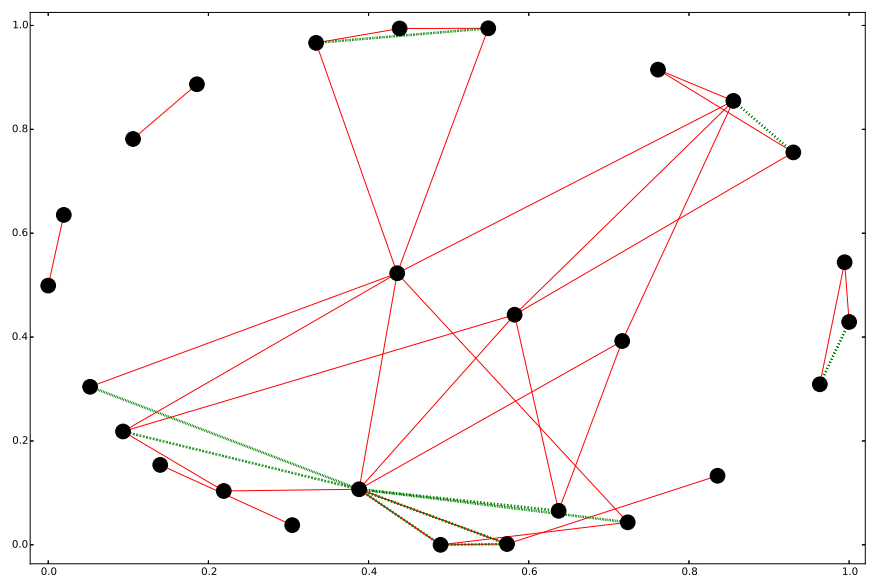

Fig. 2. Example network created from the SCAD dataset, from "Spontaneous Demonstration" conflicts in Egypt in 2014. Nodes represent actor (groups) Red links indicate at least one event performed from one node to another. Green dotted links indicate cooperation for at least one event between nodes.

accounting for inconsistent capitalisations and punctuation), highlighting wide breadth in the types of groups involved in conflict. Other extractable characteristics from the dataset include the activeness of groups (i.e., the frequency of actions performed), the concentration of their conflict (i.e., number of groups acted upon), variety of actions, and the spatial and temporal nature of their activity, through the typical location and time of the actions (where available).

Knowledge of whether actions occurred between groups can be used to synthesise a network of relationships between groups. This can be done in a number of ways. For example, negative links can be created if at least one event exists in which one group instigates a negative action towards another. Positive links are more challenging to establish. However, cooperation between groups (i.e., when multiple groups act jointly by instigating an action) can be used to provide partial insight into the positive relationships between groups. This may be observable from other sources, such as the extent to which groups naturally mix. Figure 2 shows an example graph created for a subset of the dataset to illustrate intergroup conflict, showing varying degrees of connectivity and network motifs. The properties of the resulting graph can be used as a basis to create meaningful links between groups in generative models.

Overall, this analysis indicates that the richness of the SCAD dataset enables characteristics of groups to be extracted that are relevant to generative models of group conflict, helping to inform the design choices of the parameters concerning "typical" groups. However, the quality of these characteristics is dependent on the quality of the data (e.g., correctness and completeness), as well as the temporal and spatial boundaries of groups, which can be challenging to establish.

\section{B. Inter-Group Actions}

A challenge in creating event-driven generative models concerns determining the types of actions that can occur, 
TABLE III

FREQUENCY OF THE TYPES OF EVENT CONTAINED IN THE SCAD DATASET FOR BOTH THE ORIGINAL EVENT, AND ANY KNOWN ESCALATION AFTER. ACTIONS ARE ORDERED BY AN EXAMPLE RANKING OF SEVERITY.

\begin{tabular}{llrr}
\hline ID & Type of action & \# of actions & \# of escalations \\
\hline 1 & Limited Strike & 1204 & 25 \\
2 & General Strike & 170 & 14 \\
3 & Organized Demonstration & 1342 & 43 \\
4 & Spontaneous Demonstration & 2617 & 79 \\
5 & Organized Violent Riot & 229 & 67 \\
6 & Spontaneous Violent Riot & 1957 & 833 \\
7 & Intra-government Violence & 112 & 10 \\
8 & Pro-Government Violence & 505 & 0 \\
9 & Anti-Government Violence & 817 & 50 \\
10 & Extra-government Violence & 1886 & 34 \\
\hline
\end{tabular}

TABLE IV

FREQUENCY OF ACTIONS THAT INVOLVE DIFFERENT NUMBERS OF GROUPS INSTIGATING AND RECEIVING AN ACTION.

\begin{tabular}{|l|r|r|r|}
\cline { 2 - 4 } \multicolumn{1}{c|}{} & \# instigating=1 & \# instigating=2 & \# instigating=3 \\
\hline \# receiving=1 & 9030 & 444 & 93 \\
\hline \# receiving=2 & 929 & 295 & 48 \\
\hline
\end{tabular}

including how often, at what cost to the instigator, and the impact on the receiver. While the SCAD dataset does not offer insight into the decision process behind actions or the cost of actions, it does enable analysis into a) the typical characteristics of actions, and b) the extent to which different actions are conducted by groups.

The SCAD dataset contains a variety of types of actions, as shown in Table III, along with the number of occurrences in the dataset. Individual groups only perform a subset of these, with examples shown for a subset of groups in Figure 3. This can be used to estimate the typical probabilities of actions being performed for groups with given characteristics (e.g., as defined in Section V-A). This can also include fitting to appropriate statistical distributions, which may feature as an important component in particular types of generative models (e.g., [1]). This is shown in Figure 4 where we apply Beta distribution fittings as an example, by computing the maximum likelihood estimates of the beta distribution parameters [24].

Examination of the SCAD dataset (shown in Table IV) also reveals that actions can involve can involve more than a pair of groups $(n=1809,16.7 \%)$. Additionally, while the majority of the dataset focuses on inter-group conflict, a small proportion involved the same group as the instigator and recipient $(n=302$, $2.8 \%$ ), indicating that forms of intra-group conflict could also be considered.

\section{Impact of Actions}

The impact and fallout of an action are challenging to observe as this may be obfuscated when viewed from an external perspective. This is reflected in the limited detail contained in the SCAD dataset. Nevertheless, high level insights can be made from this information. Firstly, the SCAD dataset documents the type of escalation that occurs after

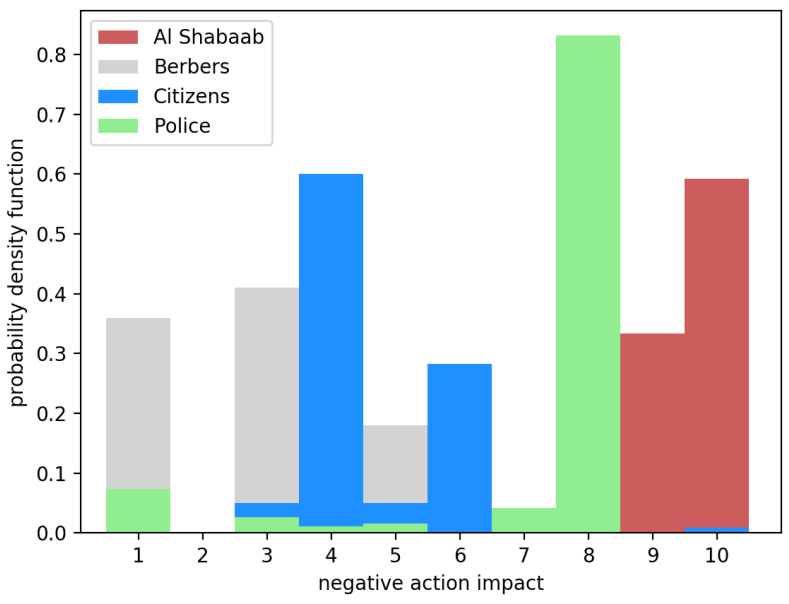

Fig. 3. An example distribution of the extent to which different types of actions are performed by a sample of groups. $\mathrm{X}$-axis values correspond to the ID column in Table III.

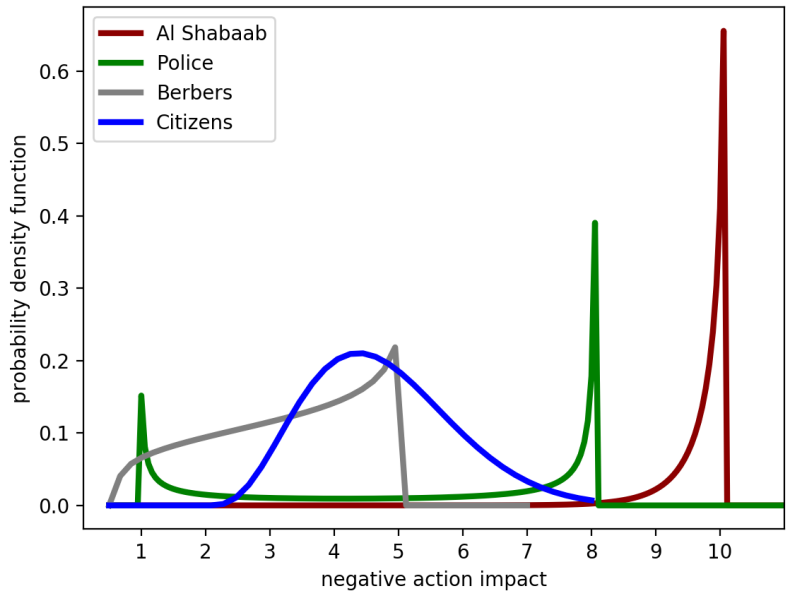

Fig. 4. An example of how the distributions in Figure 3 can be fitted through Beta distributions. X-axis values correspond to the ID column in Table III.

individual events (if any), shown in Table III. This indicates that the majority of conflict events do not result in direct escalation $(\mathrm{n}=1155,10.7 \%)$, but when this does occur, the type of escalating action is typically different from the original action $(\mathrm{n}=1126,97.5 \%)$. This is an interesting aspect against which particular generative models can be considered.

Secondly, it may be that an action results in subsequent actions, either in retaliation or as part of a pre-determined strategy or campaign. The SCAD dataset can be used to observe the frequency and nature of chains of actions. However it is challenging to define the suitable temporal and spatial boundaries of sequential actions with the limited context provided in the dataset.

\section{CONClusions And Future Work}

Generative models enable dynamics of inter-group conflicts to be examined where statistical models are not feasible due to a lack of data availability. However a challenge in building and experimenting based on these models is the choice of 
parameters and their values, with a spectrum of values often used to examine how this impacts upon a model. Despite this, the validity in terms of being representative of real-world behaviour can often not be definitively concluded. This has formed the primary focus of this paper through examining how real-world datasets (that cannot provide a complete statistical based model) can help inform the design of parameters and their values.

Firstly, we have presented a framework that characterises event-driven generative models of group conflict under three primary components: inter-group structure, inter-group actions, and the impact of these actions. From this we examine existing large-scale datasets and classify their relevance to these three components; in doing so we find that the information contained in individual datasets are typically limited to only a subset of these components, highlighting the overarching need to use multiple data-sources where possible. Finally, we selected one of these datasets and appraised its ability to provide insight into the characteristics of groups and their actions. We find that useful features relevant to group characteristics and behaviour can be extracted, however challenges remain in selecting appropriate subsets of the dataset with temporal and spatial bounds.

The analysis conducted presents several potential areas of future work. Firstly, the analysis conducted could be extended to also examine similarities and differences between different spatial regions and temporal bounds. Secondly, future work could explore how multiple conflict datasets can support data fusion, by including other datasets to augment different aspects of content (e.g., provide additional geographical semantic detail surrounding events).

Collectively this paper supports the development of generative modelling, by presenting ways in which this methodology can embody real-world data. Specifically we highlight how real-world data can be used to parameterise scenarios, to better support decision-making from generative models.

\section{ACKNOWLEDGMENT}

This research was sponsored by the U.S. Army Research Laboratory and the U.K. Ministry of Defence under Agreement Number W911NF-16-3-0001. The views and conclusions contained in this document are those of the authors and should not be interpreted as representing the official policies, either expressed or implied, of the U.S. Army Research Laboratory, the U.S. Government, the U.K. Ministry of Defence or the U.K. Government. The U.S. and U.K. Governments are authorized to reproduce and distribute reprints for Government purposes notwithstanding any copyright notation hereon.

\section{REFERENCES}

[1] D. Verma, G. Pearson, D. Felmee, A. Verma, and R. M. Whitaker, "A generative model for predicting terrorist incidents," in SPIE Defense + Security Symposium: Ground/Air Multisensor Interoperability, Integration, and Networking for Persistent ISR VIII, 2017.
[2] R. M. Whitaker, L. D. Turner, G. Colombo, D. Verma, D. Felmee, and G. Pearson, "Intra-group tension under inter-group conflict: a generative model using group social norms and identity," in Proceedings of the 8th International Conference on Applied Human Factors and Ergonomics, 2017.

[3] S. T. Fiske, "What we know now about bias and intergroup conflict, the problem of the century," Current Directions in Psychological Science, vol. 11, no. 4, pp. 123-128, 2002.

[4] T. F. Pettigrew and L. R. Tropp, "A meta-analytic test of intergroup contact theory." Journal of personality and social psychology, vol. 90, no. 5 , p. 751, 2006.

[5] M. L. Bernard, G. A. Backus, A. B. Bier, and S. F. Branch, "Behavioral influence assessment (bia): A multi-scale system to assess dynamic behaviors within groups and societies across time," in Proceedings of the 5th International Conference on Applied Human Factors and Ergonomics, vol. 5, 2014, p. 161.

[6] J. M. Epstein, Generative social science: Studies in agent-based computational modeling. Princeton University Press, 2006.

[7] E. Bonabeau, "Agent-based modeling: Methods and techniques for simulating human systems," Proceedings of the National Academy of Sciences, vol. 99, no. suppl 3, pp. 7280-7287, 2002.

[8] H. Tajfel and J. C. Turner, "An integrative theory of intergroup conflict," The social psychology of intergroup relations, vol. 33, no. 47, p. 74 , 1979.

[9] R. M. Whitaker, G. Colombo, S. M. Allen, and R. I. Dunbar, "A dominant social comparison heuristic unites alternative mechanisms for the evolution of indirect reciprocity," Scientific Reports, vol. 6, 2016.

[10] S. Reicher, "The determination of collective behaviour," Social identity and intergroup relations, pp. 41-83, 1982.

[11] D. Cunningham, S. Everton, and P. Murphy, Understanding dark networks: a strategic framework for the use of social network analysis. Rowman \& Littlefield, 2016.

[12] D. Felmlee and R. Faris, "Toxic ties: networks of friendship, dating, and cyber victimization," Social psychology quarterly, vol. 79, no. 3, pp. 243-262, 2016.

[13] I. Salehyan, C. S. Hendrix, J. Hamner, C. Case, C. Linebarger, E. Stull, and J. Williams, "Social conflict in africa: A new database," International Interactions, vol. 38, no. 4, pp. 503-511, 2012.

[14] C. Raleigh, A. Linke, H. Hegre, and J. Karlsen, "Introducing acled: An armed conflict location and event dataset special data feature," Journal of peace research, vol. 47, no. 5, pp. 651-660, 2010.

[15] R. Sundberg and E. Melander, "Introducing the ucdp georeferenced event dataset," Journal of Peace Research, vol. 50, no. 4, pp. 523-532, 2013.

[16] K. Eck, "In data we trust? a comparison of ucdp ged and acled conflict events datasets," Cooperation and Conflict, vol. 47, no. 1, pp. 124-141, 2012.

[17] S. P. Borgatti, M. G. Everett, and L. C. Freeman, "Ucinet," in Encyclopedia of Social Network Analysis and Mining. Springer, 2014, pp. 2261-2267.

[18] J. Dittrich Hallberg, "Prio conflict site 1989-2008: A geo-referenced dataset on armed conflict," Conflict Management and Peace Science, vol. 29, no. 2, pp. 219-232, 2012.

[19] L. Harbom, E. Melander, and P. Wallensteen, "Dyadic dimensions of armed conflict, 19462007," Journal of Peace Research, vol. 45, no. 5, pp. 697-710, 2008.

[20] B. Lacina and N. P. Gleditsch, "Monitoring trends in global combat: A new dataset of battle deaths," European Journal of Population/Revue Européenne de Démographie, vol. 21, no. 2, pp. 145-166, 2005.

[21] M. Fuhrmann and J. Tir, "Territorial dimensions of enduring internal rivalries," Conflict Management and Peace Science, vol. 26, no. 4, pp. 307-329, 2009.

[22] C. Raleigh and D. Kniveton, "Come rain or shine: An analysis of conflict and climate variability in east africa," Journal of Peace Research, vol. 49, no. 1, pp. 51-64, 2012.

[23] C. Raleigh and K. De Bruijne, "Where rebels dare to tread a study of conflict geography and co-option of local power in sierra leone," Journal of Conflict Resolution, p. 0022002715603767, 2015.

[24] G. J. Hahn and S. S. Shapiro, "Statistical models in engineering," in Statistical models in engineering. John Wiley \& Sons, 1968. 